\title{
Fabrication and Characterization of Single-Crystalline Au Nanowire Electrodes
}

\author{
Mijeong Kang, Hosuk Kang, Taejoon Kang, Juyoun Kwak, and Bongsoo Kim*
}

\begin{abstract}
A nanowire electrode (NWE) was fabricated using a single-crystalline Au nanowire (NW) synthesized by vapor transport method without catalysts. Au NWEs were simply fabricated by manipulating a Au NW under an optical microscope. The successful fabrication was confirmed by electrochemical oxidation and reduction of a $\mathrm{Au} \mathrm{NW}$, from which we further observed well-defined geometry and \{111\}-faceted surface of Au NWEs. We also obtained a steady-state cyclic voltammogram, indicating enhanced mass transport. The potential application of Au NWEs to drug delivery into biological substances was suggested by immobilizing DNA on Au NWEs and its electrochemical release.
\end{abstract}

\section{BACKGROUND}

$\mathrm{N}$ anoscale electric devices such as electrochemical sensors, imaging technology such as scanning electrochemical microscopy, ${ }^{1,2}$ and electrical therapeutic tools (electroporation, ${ }^{3}$ deep brain stimulator ${ }^{4}$ ) have been attracted great interests by virtue of their superior electrochemical functions, relatively facile construction and miniaturization of devices and reduced cost of material It is well known that as the dimension of electrode is reduced, electrochemical analysis is possible over localized microenvironment such as exocytosis from a single vesicle from a single cell. ${ }^{5}$ Furthermore, one can obtain various electrochemical benefits due to the reduced current resulting in reliable electrochemistry even in poorly conducting media or analysis of fast electrochemical reaction. In addition, the diffusion behavior is changed from planar diffusion to radial one so that the mass transport toward the electrode surface is enhanced and hence steady-state condition is rapidly achieved. ${ }^{6,7}$

Above many electrode materials, gold is favored owing to its chemical inertness and resultant biocompatibility and various surface modification. Recently, one-dimensional gold nanoelectrode has been widely studied. Most of the electrodes were fabricated by sharpening the macroscopic Au wire by etching or pulling or by the deposition of $\mathrm{Au}$ on other nanoelectrodes. Unfortunately, these fabrication methods require complicated multistep process. In addition, it is difficult to obtain reproducible electrode properties such as electrode dimension, surface roughness, or crystallographic

Manuscript received June 20, 2011. This work was supported by National Research Foundation of Korea Grant funded by the Korean Government (SRC Program: 2011-0001335).

All authors are with the Department of Chemistry, Korea Advanced Institute of Science and Technology, Daejeon, Korea, (corresponding author to provide phone: 82-42-350-2876; fax:82-42-350-2810; e-mail: comnsi@kaist.ac.kr). properties using these currently used fabrication methods

In this regard, we used single-crystalline Au nanowires ( $\mathrm{Au}$ NWs) to simply fabricate one-dimensional gold nanoelectrode with highly qualified electrochemical properties. In addition, the surface of a Au NWE was modified by dye-labeled DNA and the DNA was electrochemically released, suggesting potential applications of a single Au NWE to drug delivery into biological substances.

\section{MATERIALS METHODS}

\section{A. Synthesis of $A u N W$}

The Au NWs were synthesized by a simple vapor transport method without any catalysts. ${ }^{8}$ The Au slug was evaporated and the Au vapor was carried to a sapphire substrate by the flow of Ar gas. No catalysts were used, but the Au NWs were grown from the half-octahedral Au seed naturally formed on the substrate.

\section{B. Electrochemical characterization}

All electrochemical measurements were performed using an electrochemical analyzer (CHI 660D, CH instruments). The three-electrode electrochemical cell consisted of $\mathrm{Au}$ NWE, Pt wire and saturated calomel electrodes (SCE, $\mathrm{Hg} / \mathrm{Hg}_{2} \mathrm{Cl}_{2}$ electrode containing saturated $\mathrm{KCl}$ ) or mercury sulfate electrode (MSE, $\mathrm{Hg} / \mathrm{Hg}_{2} \mathrm{SO}_{4}$ electrode containing saturated $\mathrm{K}_{2} \mathrm{SO}_{4}$ ) as working, counter and reference electrodes, respectively.

\section{RESULTS}

1) Simple Fabrication of a Au NWE: A Au NWE was simply fabricated as in Figure 1. A tungsten tip (W-tip), a conducting supporter, is approached to a $\mathrm{Au}$ NW vertically grown on a sapphire substrate. The $\mathrm{Au} \mathrm{NW}$ attached to a W-tip is easily come off the substrate. Finally, W-tip is insulated with nail varnish.

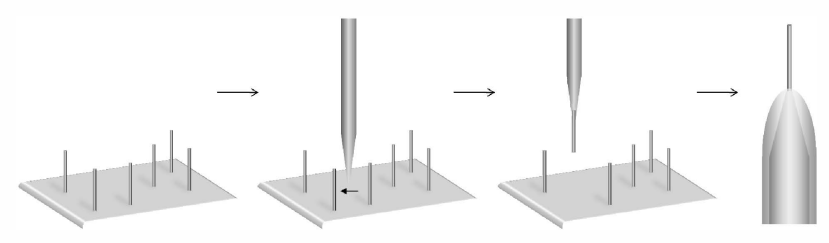

Fig. 1 Schematics showing the fabrication process of a Au NWE

2) Electrochemical Oxidation and Reduction of a Au NWE: 
Successful fabrication of a $\mathrm{Au}$ NWE was confirmed by observing the typical cyclic voltammograms (CVs) for the electrochemical oxidation of the Au NWE at $\sim 0.95 \mathrm{~V}$ and the subsequent reduction of $\mathrm{Au}$ oxide layer at $\sim 0.4 \mathrm{~V}$ in $50 \mathrm{mM}$ sulfuric acid solution (Figure 2). Unlike the Au nanoelectrode prepared by conventional methods, our Au NEW exhibited unique characteristics of single-crystalline $\{111\}$-faceted surface with the peak shape and potential.

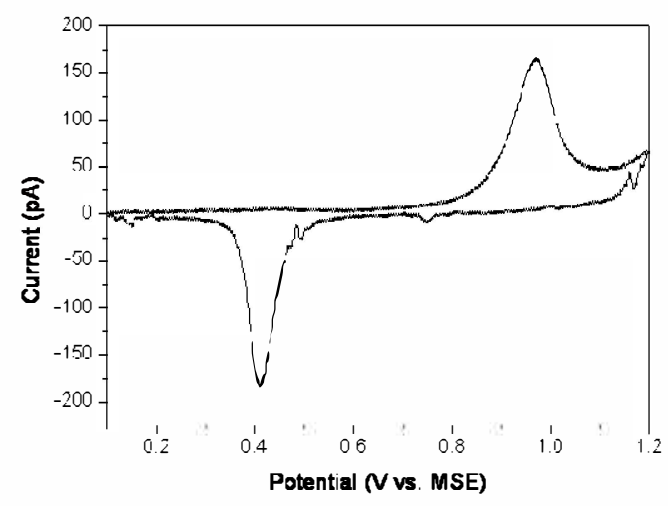

Fig. $2 \mathrm{CV}$ of a Au NWE in $50 \mathrm{mM} \mathrm{H}_{2} \mathrm{SO}_{4}$

3) Steady-State Condition at Nanoelectrode Surface: A steady-state voltammetric response as shown in Figure 3 was obtained by a $\mathrm{Au}$ NWE in the $100 \mathrm{mM} \mathrm{KCl}$ solution containing $4 \mathrm{mM}$ ferricyanide. The observed sigmoidal $\mathrm{CV}$ indicates that there is radial diffusion and thus the enhanced mass transfer of ferricyanide toward the Au NWE. Owing to this steady-state behavior, a Au NWE could be employed as a qualified nanoprobe in scanning electrochemical microscopy providing high spatial resolution originated from its small dimension.

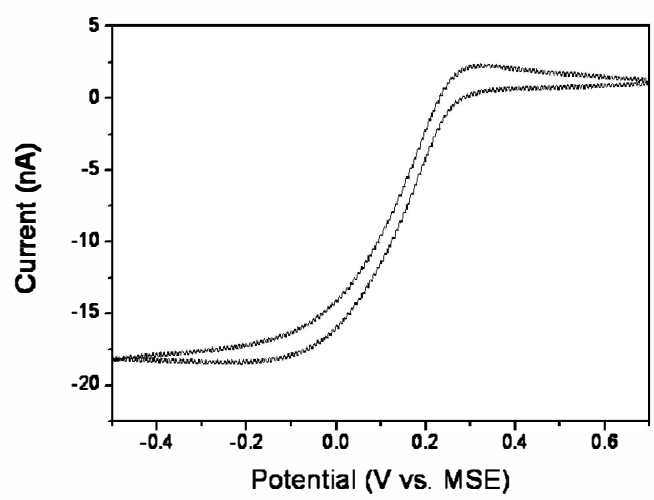

Fig. 3. CV for the reduction of ferricyanicde measured with a $\mathrm{Au}$ NWE ([ferricyanide] $=4 \mathrm{mM}$ in $100 \mathrm{mM} \mathrm{KCl}$ )

4) Electrochemical release of DNA from a $A u$ NWE: Electrochemical drug delivery has advantages such as controllable release and high delivery efficiency. Various types of drugs (DNA, protein, small molecule) can be loaded on the Au electrode surface by Au-S covalent bond. These drugs can be released by simple reductive desorption. As an example of the drugs loaded on the $\mathrm{Au}$ NWE in electrochemical drug delivery, thiolated DNA was used. In order to visualize this DNA release from $\mathrm{Au} N W$, dye-intercalated DNA was used and Au NWs after electrochemical desorption were observed by a confocal microscope. As shown in Figure 4A, the Au NEW coated with dye-labeled DNA exhibited bright fluorescence from the $\mathrm{Au}$ NEW surface. After the electrochemical potential of $-0.8 \mathrm{~V}$ (vs. SCE) was applied to the DNA-coated Au NEW, no fluorescence signal was obtained (Figure 4B) indicating successful desorption of DNA from the $\mathrm{Au}$ NWE. It is expected that this DNA-loaded Au NWE can be used to deliver DNA into a cell when it is injected into a cell and electrochemical potential is applied to release DNA.
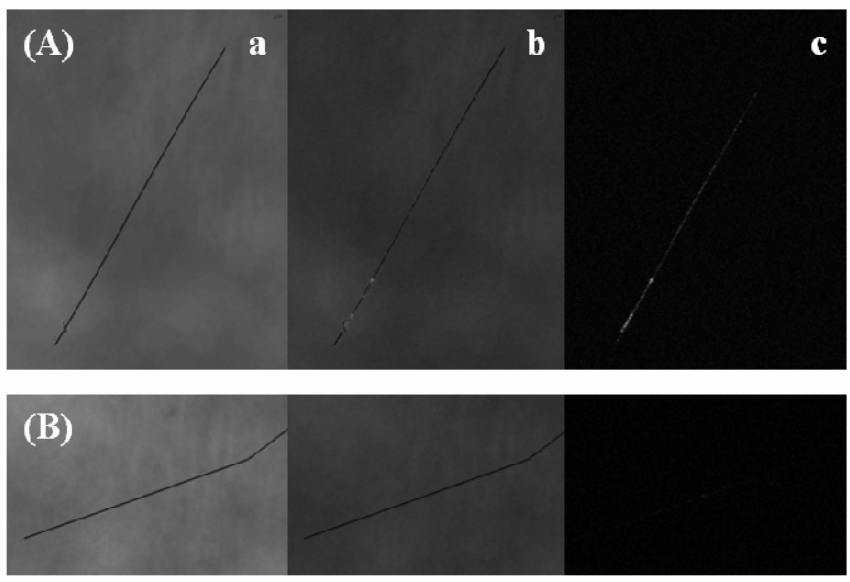

Fig. $4 \mathrm{Au}$ NWEs surface-modified with dye-intercalated double-stranded DNA before (A) and after (B) electrochemical desorption of DNA. (a: bright field, c: confocal, $b$ : emerged $(a+c)$ images $)$

\section{REFERENCES}

[1] R. M. Whiteman, "Probing Cellular Chemistry in Biological Systems with Microelectrodes." Science 2006, 311, pp. 1570-1574.

[2] I. Beaulieu, S. Kuss, J. Mauzeroll, "Biological Scanning Electrochemical Microscopy and Its Application to Live Cell Studies." Anal Chem. 2011, 83, pp. 1485-1492.

[3] T. Matsuda, C. L. Cepko, "Controlled Expression of Transgenes Introduced by in vivo Electroporation." Proc. Natl. Acad. Sci. USA 2007, 104, pp. 1027-1032.

[4] R. J. Andrews, "Nanodevices for Neuromodulation - Deep Brain Stimulation and Spinal Cord Injury." Ann. N.Y. Acad. Sci. 2007, 1122 pp. 185-196.

[5] W.-Z. Wu, W.-H. Huang, W. Wang, Z.-L. Wang, J.-K. Cheng, T. Xu, R.-Y. Zhang, Y. Chen, J. Liu, "Monitoring Dopamine Release from Single Living Vesicles with Nanoelectrodes." J. Am. Chem. Soc. 2005, 127, pp. 8914-8915.

[6] D. W. M. Arrigan, "Nanoelectrodes, Nanoelectrode Arrays and Their Applications." Analyst 2004, 129, pp. 1157-1165.

[7] D. Wei, M. J. A. Bailey, P. Andrew, T. Ryhänen, "Electrochemical Biosensors at the Nanoscale." Lab Chip 2009, 9, pp. 2123-2131.

[8] Y. Yoo, K. Seo, S. Han, K. S. K. Varadwaj, H. Y. Kim, J. H. Ryu, H. M. Lee, J. P. Ahn, H. Ihee, B. Kim, "Steering Epitaxial Alignment of Au, $\mathrm{Pd}$, and AuPd Nanowire Arrays by Atom Flux Change." Nano Lett. 2010, 10, pp. 432-438. 